\title{
The Research of the Cold Extrusion Shaping Technology of the Tubular Component with Oral Part Having Flange
}

\author{
Zhihong Yu \\ Henan Mechanical and Electrical Engineering College, Xinxiang, Henan, P.R. China 453000
}

Llyuxinhui@126.com

Key words: Cold extrusion, Flange, Tubular component, Shaping technology

\begin{abstract}
In the basis of researching the shaping methods to the tubular component with oral part having flange, the varieties of feasible forming process schemes are analyzed comprehensively under the premise of ensuring not rupture, with combination of many forming processes such as cold extrusion, cold stamping and upsetting. In the middle of cold extrusion shaping of the tubular component with oral part having flange, in the light of solid blank selected, the varieties of technology schemes that are utilized flexibly in the production are proposed and the problems existed in the schemes are compared and analysed and the corresponding prevention and improvement measures are put forward. In the production of the tubular component with oral part having flange, the cold extrusion technology is used to give the reference to a certain extent to the similar parts of forming process and die design.
\end{abstract}

\section{Introduction}

The tubular component with oral part having flange has metal parts of guide tube and sleeve cylinder such as a supporting rod and equipment base commonly and its typical structure is shown in figure 1. These type of parts can be pressworked coldly by the combination of blanking-drawing-punching-modification and stamped coldly by blanking-drawing-punching-flanging. But when the tube or cylinder is high, especially for the thin-walled tube parts, the cold stamping technology can not only reduce the production efficiency but also increase the production cost in that the number of procedures will be increased to ensure that parts will not rupture with too large tensile stress under the cold stamping forming ability limit of the plate. But the cold extrusion bulk forming process of little chip or chipless is employed which not only can improve the ratio to material used but also can reduce the machining time to improve production efficiency, and it is more important that cold extrusion forming process can obviously improve the mechanical properties of the blank material to obtain high quality tubular products. [1]So, this type of part is worked more by cold extrusion processing in production.

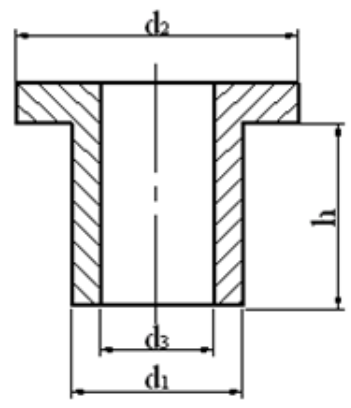

Fig.1 Diagram of part

\section{To Determine Shaping Process Scheme}

Analysis of Cold Extrusion Shaping Process of Hollow and Solid Blank. Based on the requirements on the blank proposed by cold extrusion process to form easily, that are the blank similar in shape to the product, hollow tube blank is the most suitable shape of blank[2]. For 
different size of the hollow blank, parts can be worked by a process of direct extrusion or upsetting extrusion and cold extrusion when the workpiece flange is narrow and deformation degree less than the allowable degree of deformation $\left(\varepsilon_{\mathrm{F}}<\varepsilon_{\text {Fpermission }}\right)$; intermediate cold extrusion process should be increased to reduce the deformation degree and ensure easy forming parts when the workpiece flange is wide. with the shape of parts similar to the hollow blank selected, the number of process is few and production efficiency high and die life high because inhomogeneous deformation degree of extrusion deformation is reduced to make cold extrusion forming easy. However, manufacturing of extrusion punch is difficulty in that it must be guided by mandrel in order to effectively prevent the extrusion wall cracking and defective phenomenon to ensure that qualified extrusion parts will be achieved[3].If the solid blank is chosen as the extrusion material, although its shape is not high degree similar to shape of product, the forming process is far more than a hollow blank and more capable of meeting the production flexibility and practicality in contrast with the hollow blank.

Scheme of Cold Extrusion Shaping Process of Solid Blank. When solid blank is chosen as cold extrusion processing objects to this part, in addition that these schemes are carried out that are a direct extrusion, inverted extrusion or upsetting extrusion forming before punching bottom skin under the allowable deformation degree with blank of different dimension, those schemes may be extracted which are combinations with various types of molding methods such as the cold extrusion, cold stamping and upsetting with $\varepsilon_{\mathrm{F}}>\varepsilon_{\text {Fpermission, }}$, especially with the diameter $\mathrm{d}_{0}$ of solid blank meeting the conditions of $d_{2}>d_{0}>d_{1}$. In this paper, according to the product's structure feature and metal's flow characteristic during extrusion forming process, 4 kinds of cold extrusion process schemes are designed for these solid blanks of $\mathrm{d}_{2}>\mathrm{d}_{0}>\mathrm{d}_{1}$ :

(1) Scheme one: when flange is narrow or cylinder is not deep and t of wall thickness is large with $\varepsilon_{\mathrm{F}}<\varepsilon_{\text {Fpermission, }}$, the cold extrusion forming process scheme of " upsetting extrusion-reverse extrusion-punching bottom skin " will be selected and its procedure schematic diagram is shown in figure 2 .
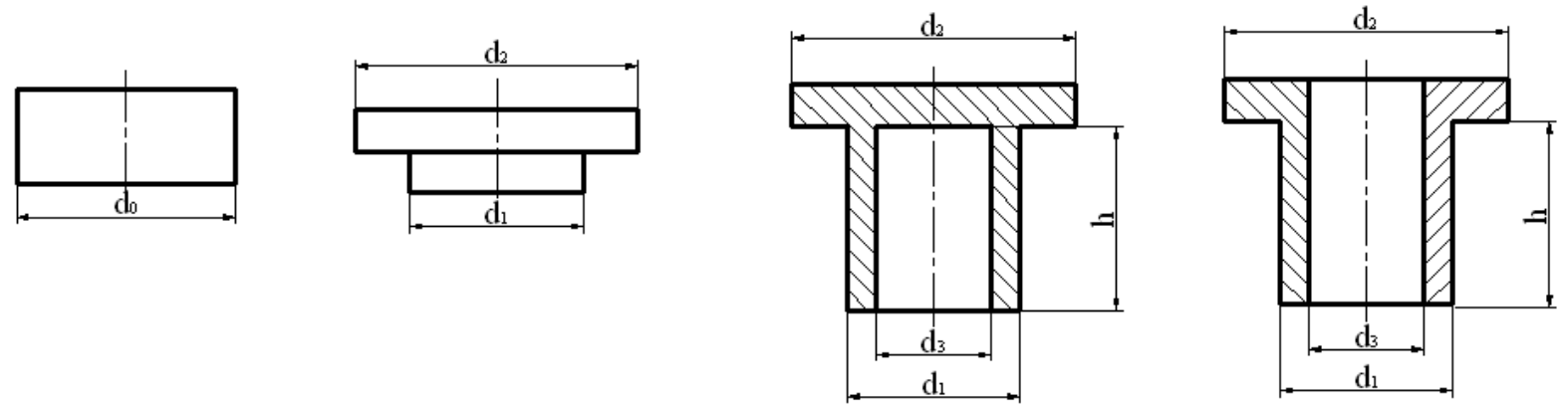
(a) blank
(b) upsetting extrusion
(c) reverse extrusion
(d) punching bottom skin

Fig. 2 The cold extrusion forming procedure diagram of the scheme one

(2) Scheme two: when flange is broad or cylinder is deep and t of wall thickness is small with $\varepsilon_{\mathrm{F}}>\varepsilon_{\text {Fpermission, }}$ the cold extrusion forming process scheme of " direct extrusion-cold upsetting-punching bottom skin " will be selected and its procedure schematic diagram is shown in figure 3.

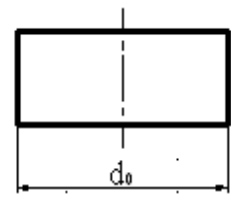

(a) bla

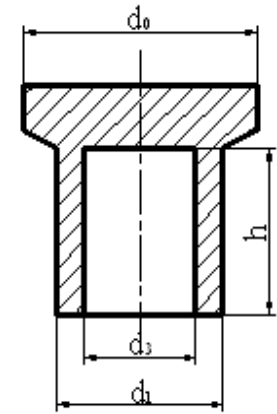

(b) direct extrusion

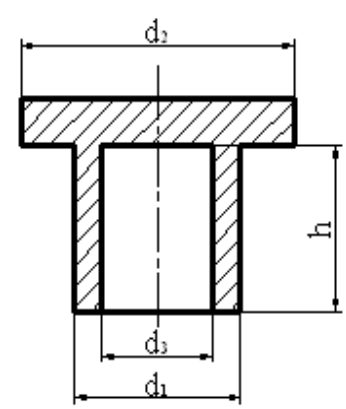

(c) cold upsetting

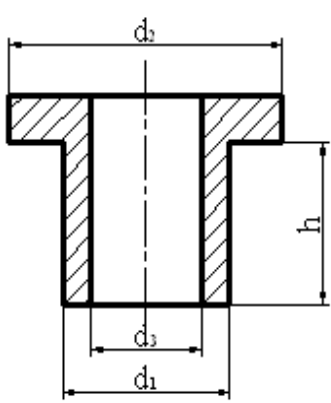

(d) punching bottom skin

Figure. 3 The cold extrusion forming procedure diagram of the scheme two 
(3) Scheme three: when the deformation degree $\varepsilon_{\mathrm{F}}$ is $\varepsilon_{\mathrm{F}}>\varepsilon_{\mathrm{Fpermission}}$, according to the metal in the rigid translational zone only translating rigidly and changing shape no longer, the cold extrusion forming process scheme of " combined extrusion-reverse extrusion-punching bottom skin-upsetting " will be selected[3] and its procedure schematic diagram is shown in figure 4.

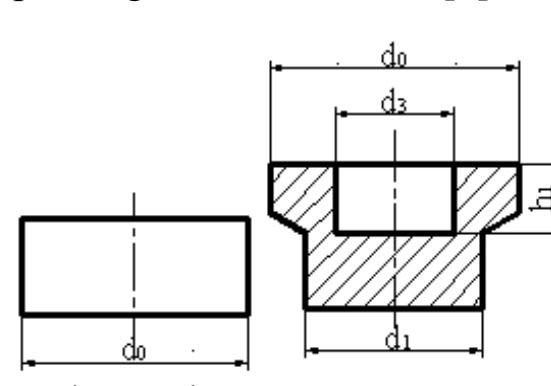

(a) blank

(b)combined extrusion
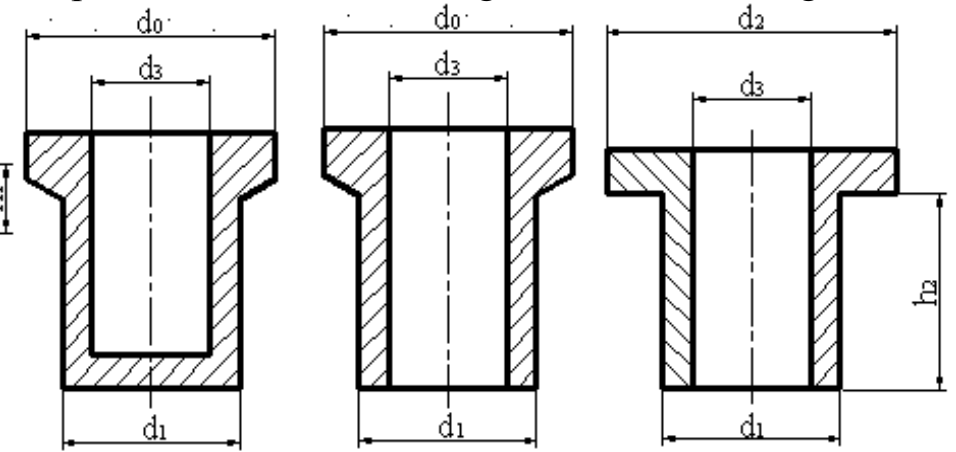

(c) reverse extrusion (d)punching bottom skin

(e) upsetting

Figure. 4 The cold extrusion forming procedure diagram of the scheme three

(4) Scheme four: when flange is thin and broad or cylinder is very deep and t of wall thickness is very small with $\varepsilon_{\mathrm{F}}$ over $\varepsilon_{\mathrm{Fpermission}}$ far, the deformation degree of the cold extrusion forming should be reduced through the process scheme of " reverse extrusion-punching bottom skin-direct extrusion-upsetting ". Its procedure schematic diagram is shown in figure 5.
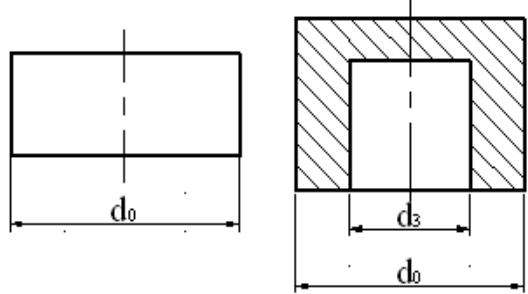

(b) reverse extrusion

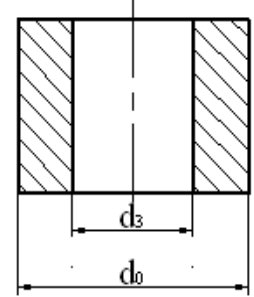

(c) punching bottom skin

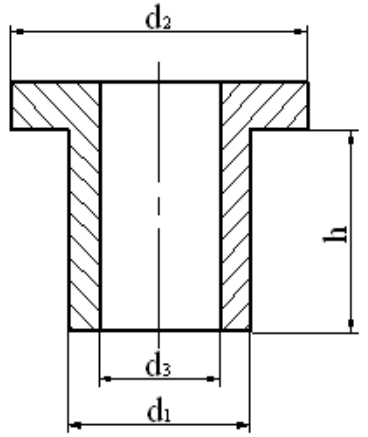

(d) direct extrusion

(e) upsetting

Figure. 5 The cold extrusion forming procedure diagram of the scheme four

\section{Scheme Analysis and Discussion}

In the scheme one, it should be paid attention that the metal in flange will not participate in deformation during reverse extruding cylinder wall after upsetting deformation and the size of cylinder depth should be the same as the height of round column after backward extrusion, otherwise the flange will present these process problems such as partially forming and necking and bulging in porthole. The defects of this scheme are that the flange metal's forming dimensional requirements and locating the flange metal in waiting deformation area completely in reverse extrusion are hard to control in upsetting extrusion.

In the scheme two, what we should pay attention to is that the hole depth should be equal to the height of round column after forward extrusion forming, otherwise, process problems such as necking and bulging will occur in porthole ;in addition, the sequence of the procedures between the forward extrusion process and cold upsetting process should not be inverted, otherwise, due to the different two adjacent cross-sections more apparent, flange metal is transferred to the cylinder wall through too much flow distance to cause serious turbulent in deformation process , and then crease will occur and dehiscence may even occur in transition location. The defects of this scheme are that the mandrel that guarantees the hole shape is easily to buckle and surface crease occurs easily in cylinder wall to influence the parts quality in cold upsetting [5]. 
In the scheme three, it should be paid attention that the hole depth should be equal to the flange depth after combined extrusion forming, and the flange should be guaranteed to be in the rigid transition area when reverse extruding cylinder wall, or if flange part is also involved in deformation, the deformation degree will be led to increase and extrusion forming is not easy; in addition, the sequence of the procedures between the punching bottom skin and upsetting should not be inverted, or mandrel will be caused to break probably. The drawbacks of this scheme are that the procedure number is too many, deformation degree of combined extrusion is big, and annealing-process should be added necessarily, so the production efficiency must be greatly reduced and additionally, the middle dimension and diversion surface are more difficult to design.

In the scheme four, the phenomenon that mandrel is easily to break is avoided in upsetting; When cylinder wall is formed by direct extruding, relationship between the cylinder height and hole depth is no need consider. Additionally, even though the number of procedure is too many, annealing-process is unnecessary to be added and production efficiency will not be too low.

For cylinder parts with wider flange, depending on the procedure above, sizes of $d 1$ and $d_{2}$ are not generally formed in a combined forming in upsetting extrusion, and the procedure number should be increased and upsetting procedure is often after cold extrusion process; Of course, we can firstly cold upsetting $d_{2}$, and then add a procedure to direct extrude or reverse extrude $d_{3}$ and $d_{1}$ when cylinder depth is too deep. These schemes also apply to these applications where dimension of blank is $\mathrm{d}_{0}=\mathrm{d}_{2}$ or $\mathrm{d}_{0}=\mathrm{d}_{1}$ and part's flange is wider, cylinder depth is deeper and wall thickness is thinner.

\section{Summary}

Several schemes of the same parts can be obtained by choosing different blank and using different forming method. Under the same conditions, where $\varepsilon_{\mathrm{F}}$ is permitted and parts quality can be ensured, process sequence can be properly adjusted or organically combined to get more flexible and practical solutions. However, the number of procedure should not be designed too few, or the deformation degree will be too big and the punch will easily destabilize, and this will go against forming; [4] Likewise, that the number of procedure should not be too many, or the middle procedures are too many, especially annealing-in-process increased, will reduce the production efficiency and the quality of the product.

\section{Reference}

[1] R.J.Xu:Shanxi Coking Coal Science \& Technology, (2006) No.6 p.35 (In Chinese)

[2] D.M.Zhai: Cold Extrusion Technology and Die Design [M]. (Chemical Industry Press, China 2004), 2004 (In Chinese)

[3] S.Z.Zhang: Mechanical Bearing (2006)No.2 p.16 (In Chinese)

[4] Z.H.Yu:Henan Mechanical and Electrical Engineering College Journal,(2007).No.5 p.6 (In Chinese)

[5] L.L.Jia: Extrusion Technology and Die [M] (Machinery Industry Press, China 2004)(In Chinese) 